\title{
Nadi Yantra: a robust system design to capture the signals from the radial artery for assessment of the autonomic nervous system non-invasively ${ }^{*}$
}

\author{
Abhinav ${ }^{1}$, Meghna Sareen ${ }^{1}$, Mahendra Kumar ${ }^{1}$, Jayashree Santhosh ${ }^{2}$, \\ Ashok Salhan ${ }^{3}$, Sneh Anand ${ }^{1}$ \\ ${ }^{1}$ Centre for Biomedical Engineering, Indian Institute of Technology, Delhi, India; ${ }^{2}$ Computer Services Centre, Indian Institute of \\ Technology, Delhi, India; ${ }^{3}$ Defence Institute of Physiology \& Allied Science, Defence Research and Development Organisation, \\ Delhi, India. \\ Email: ${ }^{1}$ abhinav_cbme_iitd@hotmail.com; ${ }^{2}$ meghnasareen@gmail.com
}

Received 15 June 2009; revised 30 June 2009; accepted 1 July 2009.

\begin{abstract}
Ayurvedic and other alternative medical practitioners throughout the world have been using pulse diagnosis to detect disease and the organ at distress by feeling the palpations at three close yet precise positions of the radial artery. This paper presents a robust electro-mechanical system, 'Nadi Yantra' which uses piezoelectric based pressure sensors to capture the signals from the radial artery. Morphology of the waveforms obtained from our system concurs with standard physiological arterial signals. Reproducibility and stability of the system has been verified. Signal processing techniques were applied to obtain features such as amplitude, power spectral density, bandpower and spectral centroid to reflect variations in signals from the three channels. Further, wavelet based techniques were used to process the pressure signals and percussion peaks were identified. The interval between the percussion peaks was used to calculate Heart Rate Varibility (HRV), a useful tool for assessing the status of the autonomic nervous system of the human body noninvasively. Time domain indices were calculated from direct measurement of peak-peak (PP) intervals and from differences between the PP intervals. Frequency domain indices such as very low frequency (VLF) power, low frequency (LF) power, high frequency (HF) power, LF/HF ratio were also calculated. Thereafter, nonlinear Poincare analysis was carried out. A map of consecutive PP intervals was fitted to an ellipse using least squares method. Results from 7 datasets are depicted in this paper. A novel pressure pulse recording instrument is deve-
\end{abstract}

"Nadi Yantra has been applied for patent (pending approval); Nadi stands for Pulse and Yantra means Instrument. loped for the objective assessment of the ancient science of pulse diagnosis. The features calculated using multi resolution wavelet analysis show potential in the evaluation of the autonomic nervous system of the human body.

Keywords: Radial Artery; Pulse Diagnosis; Power Spectral Density; Spectral Centroid; Multi Resolution Wavelet; Autonomic Nervous System; Heart Rate Variability; Time Domain; Frequency Domain; Poincare

\section{INTRODUCTION}

In ancient literatures of the Ayurveda, Chinese, Unani, and Greek medicine, pulse based diagnosis has its own unparalleled importance. The organ under distress is zeroed down by feeling the palpations from the three fingers (index, middle and ring) placed on the radial artery (Figure 1). These pulsations dictate the physiological status of the entire human body [1]. This is a tedious and highly subjective process and takes years of practice to master this art [2].

Pulse has been ubiquitously accepted by modern clinicians as well. They examine the pulse using the method of trisection i.e. apply pressure until the pulse is maximal, and then vary pressure while concentrating on the phases of the pulse. The arterial pulse variants (for example pulsus alternans, bisferiens pulse, bigeminal pulse) are used in detecting cardiac disorders. However, alternative medicine practitioners carefully examine pulses at different depths, each connected with a specific part of the body and each believed to register even the slightest physiological based change.

If there can be a device that can give an objective assessment of the science of pulse diagnosis, it will assist disease diagnosis noninvasively. It will be used by alternative medicine practitioners as well as modern clinicians. 
Analysis of the R-R time series has been commonly used in electrocardiographic (ECG) signals. ECG signals are electrical signals of the heart and pressure signals from the radial artery are mechanical signals. However, pressure signals also show significant complexes just like the QRS of ECG waves. Therefore, a similar analysis on percussion peak-peak (PP) time series can be carried out to provide information regarding the status of the autonomic nervous system noninvasively.

In this paper we discuss our device Nadi Yantra, the three sensors of which simulate the human fingers. In Section 2 the instrument has been explained in detail. Section 3 describes the wavelet based signal processing for calculation of heart rate variability and features for evaluation of the autonomic nervous system noninvasively. Section 4 describes experiments conducted. Inferences are drawn in Section 5.

\section{THE INSTRUMENT-NADI YANTRA}

There is a need for the development of a quantitative system for pulse diagnosis [3]. Investigations have been attempted globally to develop a system that replicates the human three-finger method of pulse based diagnosis [4,5]. In previous attempts

1) Signals have been captured for a very small span of time (1-2 minutes).

2) External pressure applied over the sensors varies while recording.

3) Motion artifacts become a reasonable consideration when the recordings are for a longer duration of time.

Advances over the earlier systems are that Nadi Yantra allows recording for hours by an automated external pressure on the three positions thereby completely removing the potential for errors incurred when pressure is applied (Figures 2, 3, 4). The locking mechanism significantly resists the motion artifacts as well.

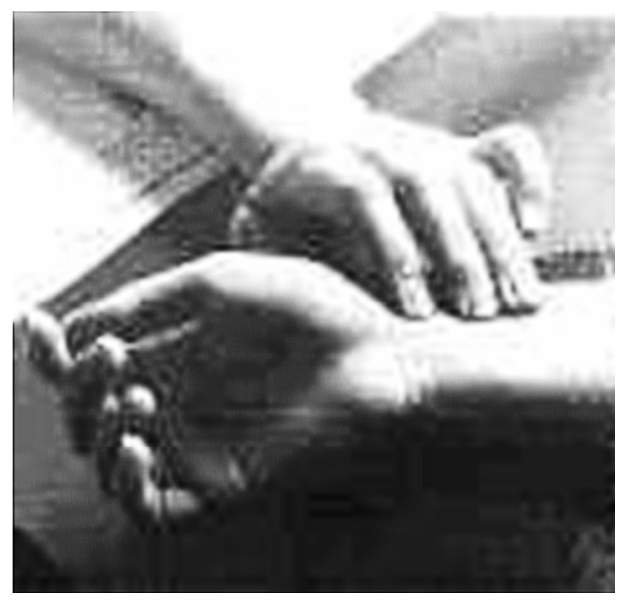

Figure 1. A practitioner evaluating the patient's pulse.

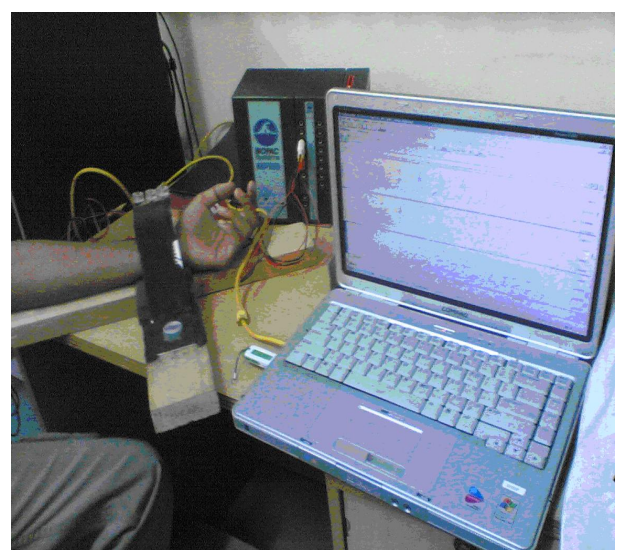

Figure 2. Recording the Pulse using Nadi Yantra.
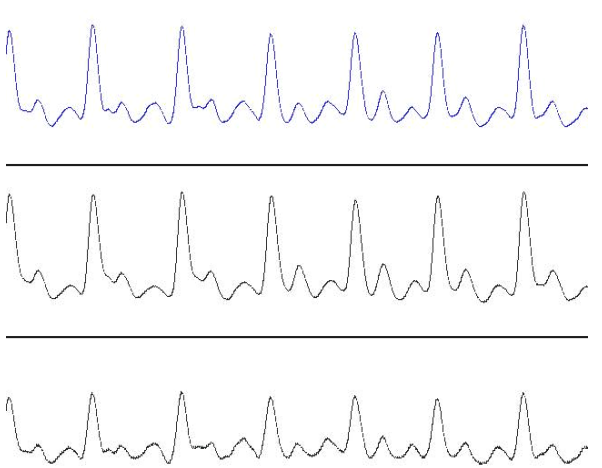

Figure 3. Signals (zoomed) as observed in the three channels.

In the mechanical design, the system has three finger like projections whose positions can be adjusted at the tip region to find out the best locations to capture the signal. Springs attached to them help in damping thus simulating the natural damping present due to muscles in the tip region of the practitioner's fingers (Figure 5).

Once the three best positions are found, they are locked with another hard spring fitting. This lock resists the motion artifacts as well. Discrete increments in pressure are possible by changing the lock's position towards the slant side. (Figure 6).

In the electrical design, we used three identical piezo film based sensors to capture the waveform. The raw signal was filtered, amplified, and transferred to the computer using BioPac $150^{\mathrm{TM}}$ (Signal Acquisition System) operated at a sampling rate of 1000 samples per second.

It is found that the signal captured using Nadi Yantra corresponds well with standard pulse from the radial artery (Figure 7, Figure 8). A standard pulse from the radial artery comprises of the following waves: [5]
a) Percussion Wave
b) Tidal Wave
c) Dicrotic Wave
d) Valley 


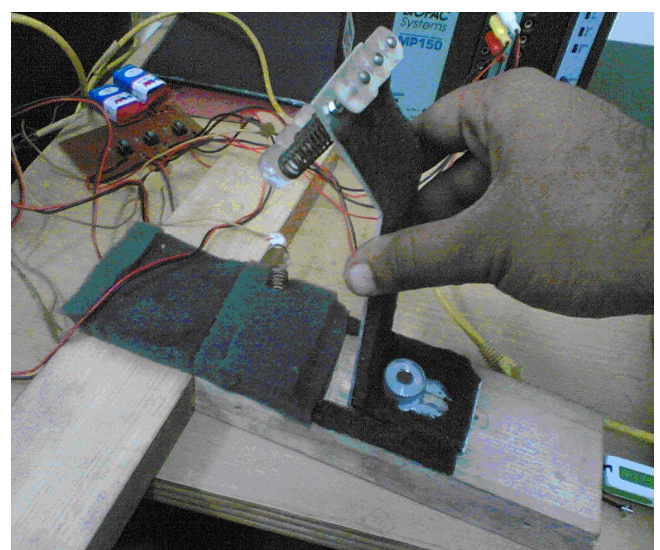

Figure 4. Flexibility of the system.

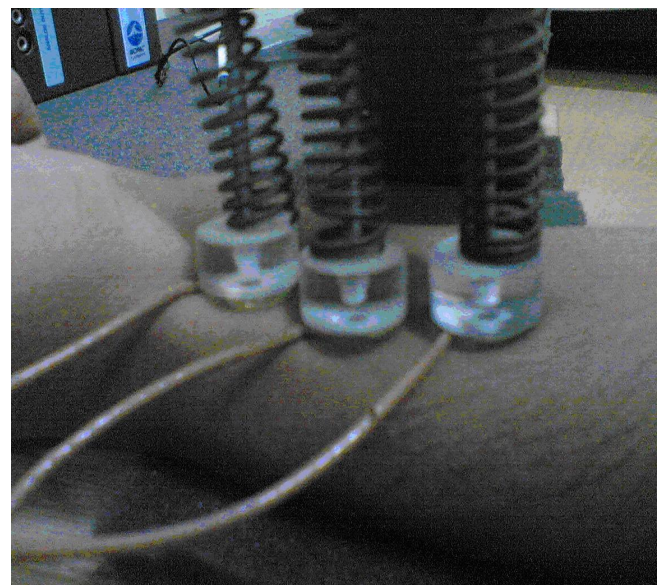

Figure 5. Simulation of fingers with the pressure sensors.

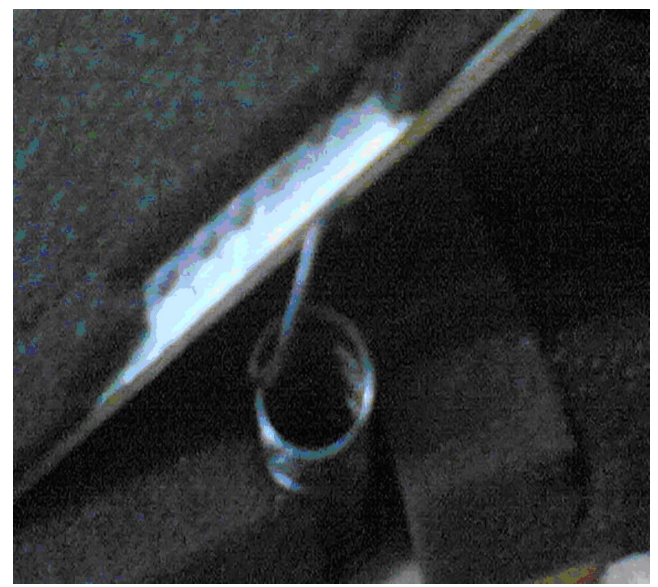

Figure 6. Lock mechanism for discrete pressure increment.

\section{ANALYSIS OF RADIAL PULSE SIGNAL}

\subsection{Pressure Wave Decomposition Using Wavelets}

A radial pulse signal feature extraction system using wavelet-based multi-resolution analysis was developed and evaluated.
The choice of the wavelet function depends on how closely the scaling function matches the shape of the original signal [6,7]. Daubechies 9 (D9) of Daubechies is similar in shape to the radial pulse signal complex and was used in the decomposition.

Figure 9 shows the details of a signal captured by Nadi Yantra. The original signal is shown at the top of the plot. Below the signal, the details for seven wavelet scales are shown which are scaled for better illustration. Adding together all these details plus the signal approximation A7 returns the original signal.

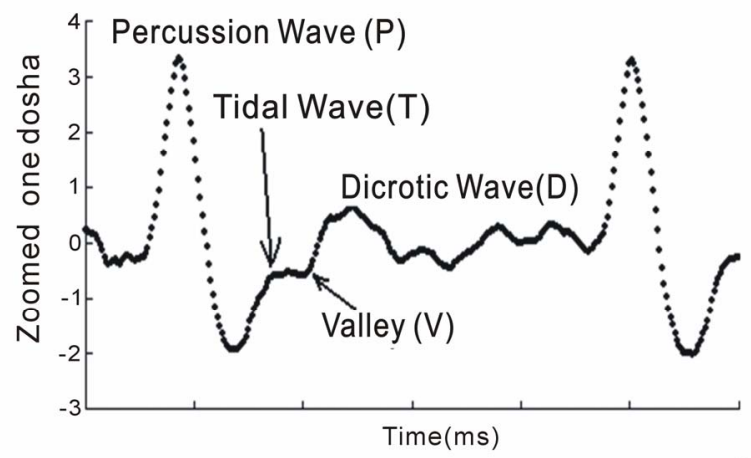

Figure 7. A standard signal from the radial artery.

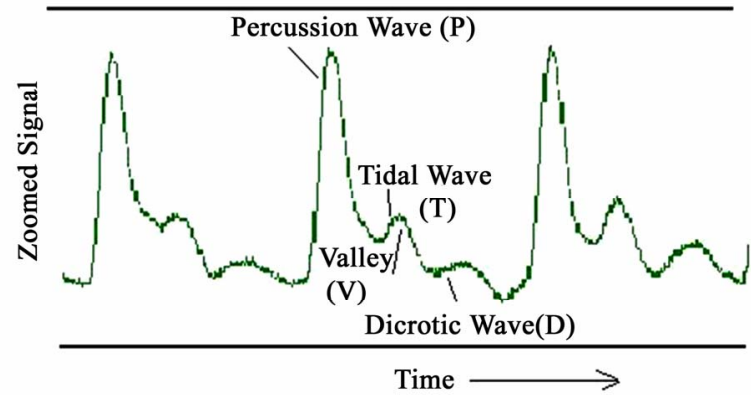

Figure 8. Zoomed signal as acquired by Nadi Yantra. (Note: No digital filters have been used to capture these raw signals)

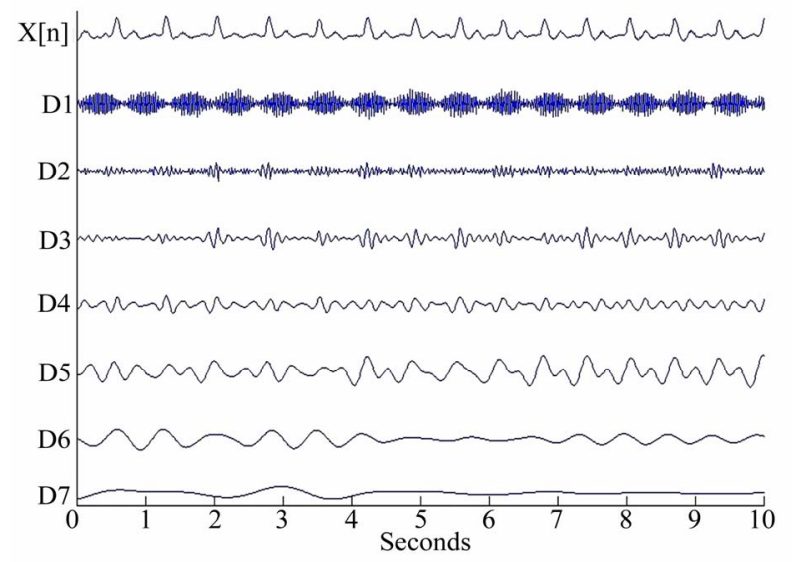

Figure 9. Wavelet decomposition of a typical radial pulse signal. 
A few points can be noted from the plot.

1) The high frequency noise content is captured at the smallest scales, namely D1, D2 and D3.

2) Scales D4, D5 and D6 contain most of the information that depicts the radial pulse signal.

3) Also, scales D7 (and above) correspond to very low frequencies which do not contribute to the complex. They account for the DC component of the signal and baseline wander resulting from motion etc.

\subsection{Preprocessing of Signal}

Low frequencies (which constitute baseline wander) appear at high scales (details D7 and above). Removal of such details corrects baseline wander. High frequency burst of noise is captured at the smallest scales (which correspond to high frequencies), namely D1, D2 and D3. Removal of such details corrects noise. Additionally, a notch filter was applied to remove the $60 \mathrm{~Hz}$ power line interference. The result is shown in Figure $\mathbf{1 0 .}$

A frequency domain analysis of the denoised pressure signal was carried out. A Fourier Transform of the pressure signal from the radial artery is shown below. It shows the significant frequencies present in the signal. The signal contains low frequencies from $0-10 \mathrm{~Hz}$ (Figure 11).

\subsection{Percussion Peak Detection}

It was observed that the details D3, D4 and D5 are the most significant and contain the information that represent the radial pulse signal complex, including percussion wave. These details are devoid of high frequency noise and low frequency baseline wander as well as mean DC component. The signal was appropriately squared to enhance percussion peaks which ensured better detection. A thresholding technique was used to detect the percussion peaks. Thereafter, the peak-peak (PP) time series was obtained.

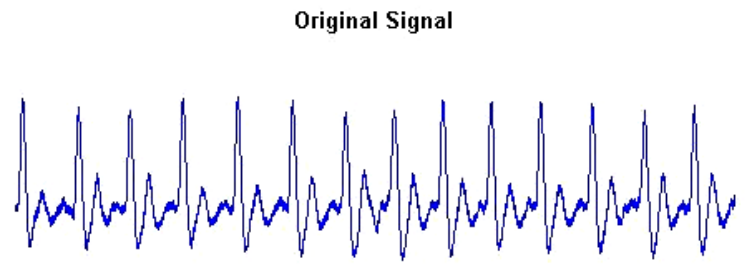

Notch Filtered Signal

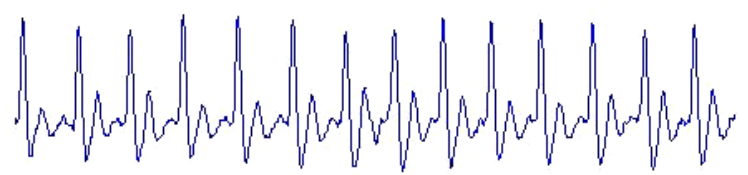

Figure 10. Clean denoised signal.

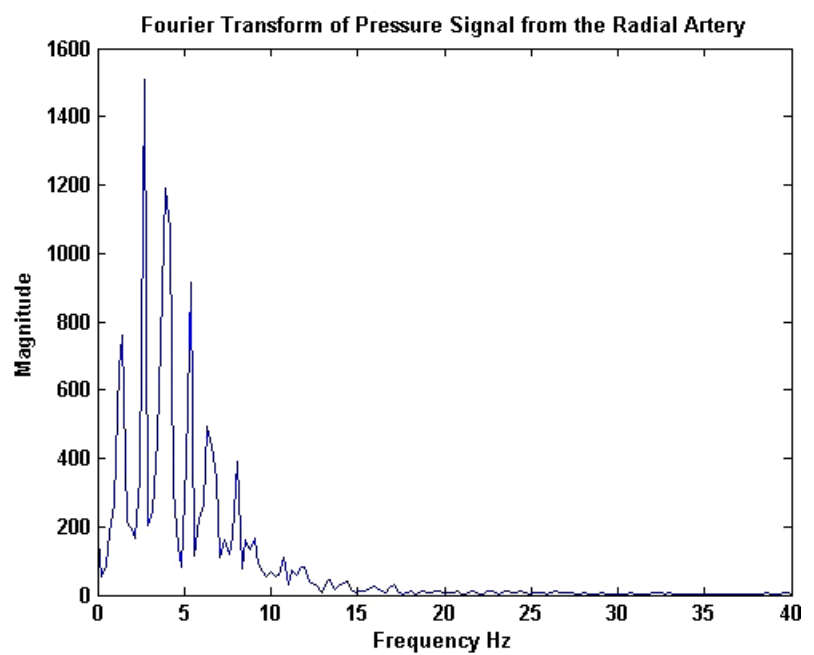

Figure 11. Fourier transform of denoised pressure signal.

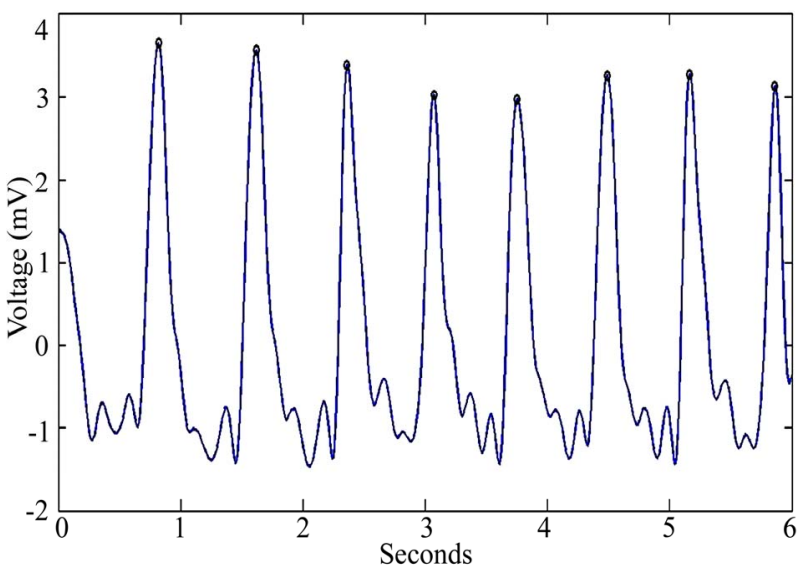

Figure 12. Peak detection in pressure signals using wavelets.

\subsection{Feature Extraction from Percussion Peak-Peak (PP) Intervals}

It is well known that perturbations to autonomic activity, such as respiratory sinus arrhythmia and vasomotor oscillations cause corresponding fluctuations in heart rate. The alterations of the heart beat known as heart rate variability (HRV) is a useful tool for assessing the status of the Autonomic Nervous System (ANS) non-invasively [8,9]. Our approach for HRV calculation is based on Percussion complex detection. HRV signal is computed from the time difference between two consecutive percussion complexes known as the PP time series. A HRV signal is shown in Figure 13 below.

Changes in heart rate occur as a result of the autonomous nervous system's actions through the parasympathetic (P) and the sympathetic (S) pathways which have opposite influences. The $\mathrm{S}$ stimulation leads to an increase in heart rate and the $\mathrm{P}$ stimulation does the opposite. These different actions result in fluctuations in the 


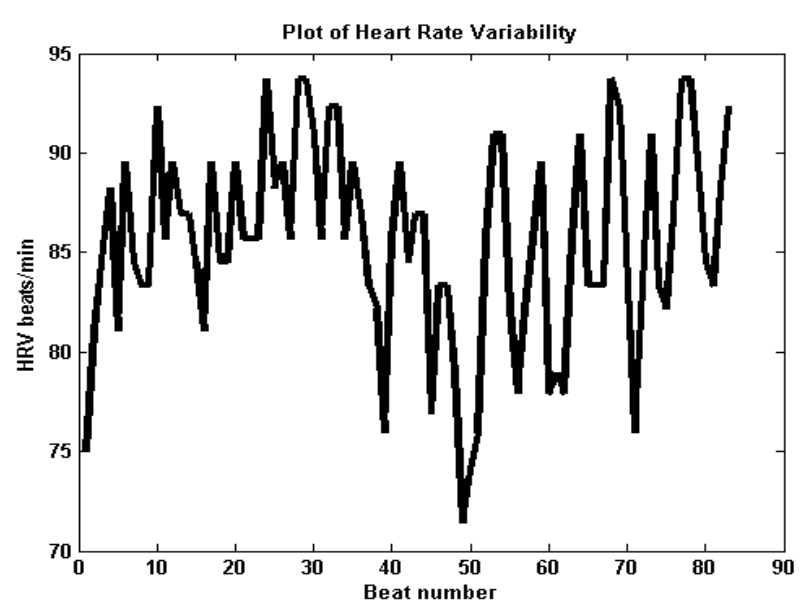

Figure 13. HRV signal.

heart rate, the best known being the sinus respiratory arrhythmia modulated by the $\mathrm{P}$, and the ones related with the baroreflex action modulated by the $\mathrm{S}$.

Time domain indices were derived from direct measurements of the PP intervals or from differences between the PP intervals. The former include mean and standard deviation (SDNN i.e. standard deviation of the normal to normal interval i.e. square root of variance), SDANN (standard deviation of the average $\mathrm{NN}$ interval calculated over short periods). The most commonly used measures derived from interval differences include RMSSD, the square root of the mean squared differences of successive PP intervals, NN50, the number of interval differences of successive NN intervals greater than 50 milliseconds and pNN50, the proportion derived by dividing NN50 by the total number of NN intervals [10]. These provide information on the short-term and long-term variability of the P-P time series.

Frequency domain indices provide information on both total variability as well as distribution as a function of frequency. The spectral analysis of this HRV signal allows to quantitatively distinguish between the different activities of the ANS such as very low frequency component (VLF: 0-0.04 Hz), a low frequency component (LF: 0.04-0.15 Hz) and a high frequency component (HF: $0.15-0.4 \mathrm{~Hz}$ ). HF power is supposedly a pure measure of parasympathetic activity and represents momentary respiratory influences on heart rate or respiratory sinus arrhythmia, and LF power is reflective of sympathetic modulation and parasympathetic tone. It derives from short term regulation of blood pressure.

A total of 40 signals were analyzed and results from 7 datasets are shown below in Table 1 .

There is some evidence for the involvement of nonlinear phenomena in the genesis of HRV. It is conceived that assessment of HRV with nonlinear measures may supply information different from and additional to that derived through linear measures. A non-linear Poincare analysis was carried out for a representative P-P time series where a Poincare plot is a scatter-plot of the current P-P interval against the preceding $\mathrm{P}-\mathrm{P}$ interval. The $\mathrm{PP}_{\mathrm{i}}$ is plotted versus $\mathrm{PP}_{\mathrm{i}-1}$ and the plot shown below is obtained. An ellipse was fitted to it using least squares method and values of the centre of the ellipse, major and minor axis, angle with the $\mathrm{x}$ axis and equation of the ellipse obtained. The plot provides summary information as well as detailed beat-to-beat information on the behavior of the heart. A distinct advantage of Poincare plots is their ability to identify beat-to-beat cycles and patterns in data that are difficult to identify with spectral analysis [11]. The Poincare plot (Figure 14) allows the HRV researcher to measure the variability of heart rate from different points of view such as long-term variability, overall variability, variability on basal heart rate, variability on accelerated heart rate, variability on decelerated heart rate as well as the sympathetic-parasympathetic balance.

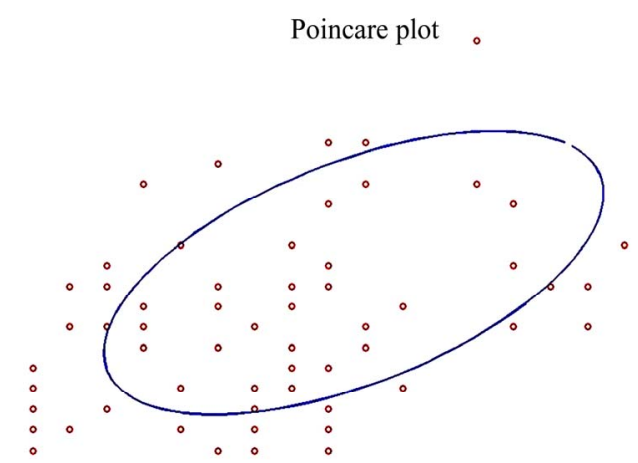

Figure 14. Poincare analysis.

Table 1. Features extracted from P-P time series.

\begin{tabular}{|c|c|c|c|c|c|c|c|}
\hline VLF (0-0.04 Hz) $* 10^{5}$ & 3.89 & 3.45 & 3.82 & 3.67 & 4.09 & 4.09 & 4.03 \\
\hline LF (0.04-0.15 Hz) $* 10^{4}$ & 1.66 & 1.38 & 2.02 & 1.55 & 1.80 & 1.81 & 1.91 \\
\hline HF (0.15-0.4 Hz) $* 10^{3}$ & 5.47 & 3.59 & 6.90 & 4.71 & 5.71 & 5.53 & 6.93 \\
\hline LF/HF & 3.03 & 3.84 & 2.93 & 3.30 & 3.15 & 3.26 & 2.76 \\
\hline Mean & 70.4 & 61.2 & 68.6 & 65.8 & 73.6 & 73.6 & 72.7 \\
\hline SDNN & 4.40 & 2.61 & 9.24 & 3.21 & 3.73 & 3.72 & 7.07 \\
\hline RMSSD & 4.51 & 2.72 & 9.63 & 3.51 & 3.97 & 3.97 & 7.38 \\
\hline SDNN/RMSSD & 0.97 & 0.96 & 0.96 & 0.91 & 0.94 & 0.94 & 0.96 \\
\hline NN50 & 69 & 84 & 73 & 79 & 72 & 72 & 72 \\
\hline pNN50 (\%) & 83.1 & 86.6 & 84.8 & 87.7 & 90 & 90 & 88.8 \\
\hline
\end{tabular}


$\mathrm{X}_{\mathrm{c}}=72.68, \mathrm{Y}_{\mathrm{c}}=72.65, \mathrm{~A}=8.55, \mathrm{~B}=4.54, \mathrm{Phi}=0.81$ where $X_{c}$ and $Y_{c}$ are the $x$ and $y$ axis centre of the ellipse, $\mathrm{A}$ and $\mathrm{B}$ are the major and minor axis of the ellipse respectively, Phi is the radian angle of the major axis with respect to the $\mathrm{x}$ axis. The equation of the ellipse is $0.6 x^{2}-0.6 x y+0.5 y^{2}-37.4 x-33.4 y+2554.7$

\section{EXPERIMENTS}

\subsection{Description of Experiment}

We chose 5 healthy volunteers to carry out a whole day analysis. The dynamics of the physiological signals were examined for pre-lunch and post-lunch states.

The control used in our analysis was the 20 sets of signals captured from the same subject over a period of time prior to lunch. The post lunch signals were compared with the control signals and the results are shown below (Figures 15-17). Signals were recorded at 1245 hrs, 1315 hrs, 1355 hrs and 1705 hrs where lunch was administered at 1420 hrs.

It can be observed that the amplitude of the $1^{\text {st }}$ channel rises steadily prior to lunch and then falls post lunch. It can also be seen that the amplitude of the signals from the $2^{\text {nd }}$ and $3^{\text {rd }}$ channels rises post lunch (Figures 15-20).

\subsection{Signal Processing and Results}

Power spectral density of the signals was calculated and plotted in Figures 21-23.

It can be seen from the cumulative PSD plots that the signals from the three channels have the same frequency components in pre-lunch and post-lunch signals. The frequency peaks are more in number in the signals before lunch as compared to that after lunch. The magnitude

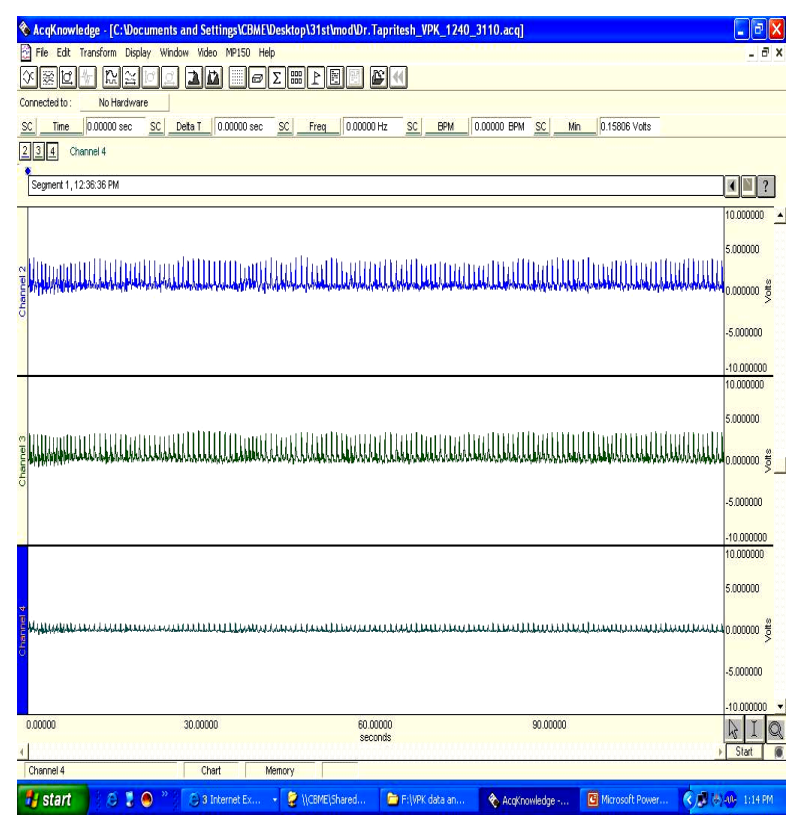

Figure 15. Signals captured at 1245 hrs.

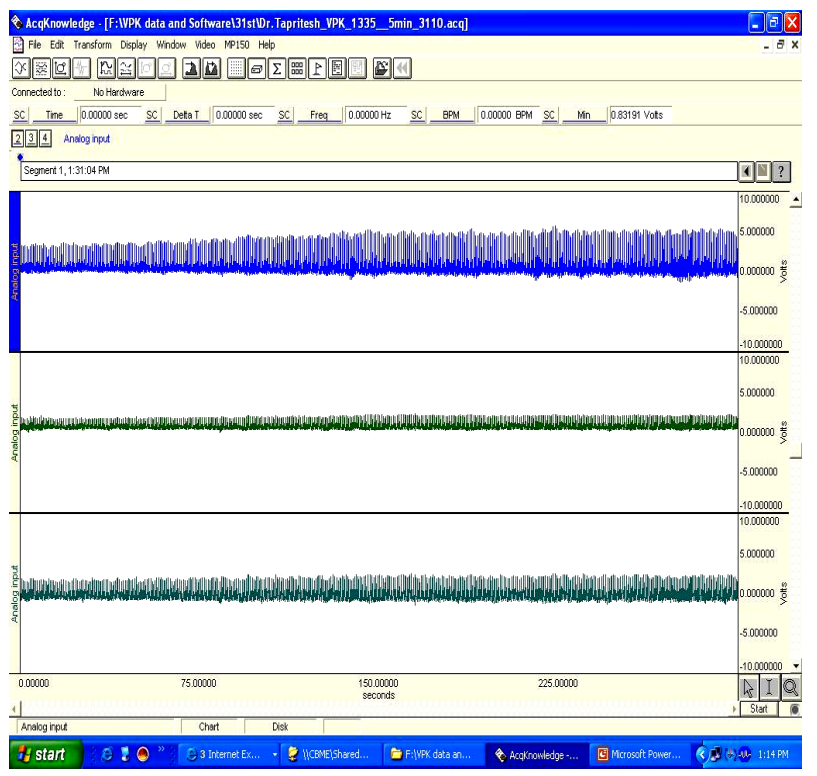

Figure 16. Signals captured at 1315 hrs.

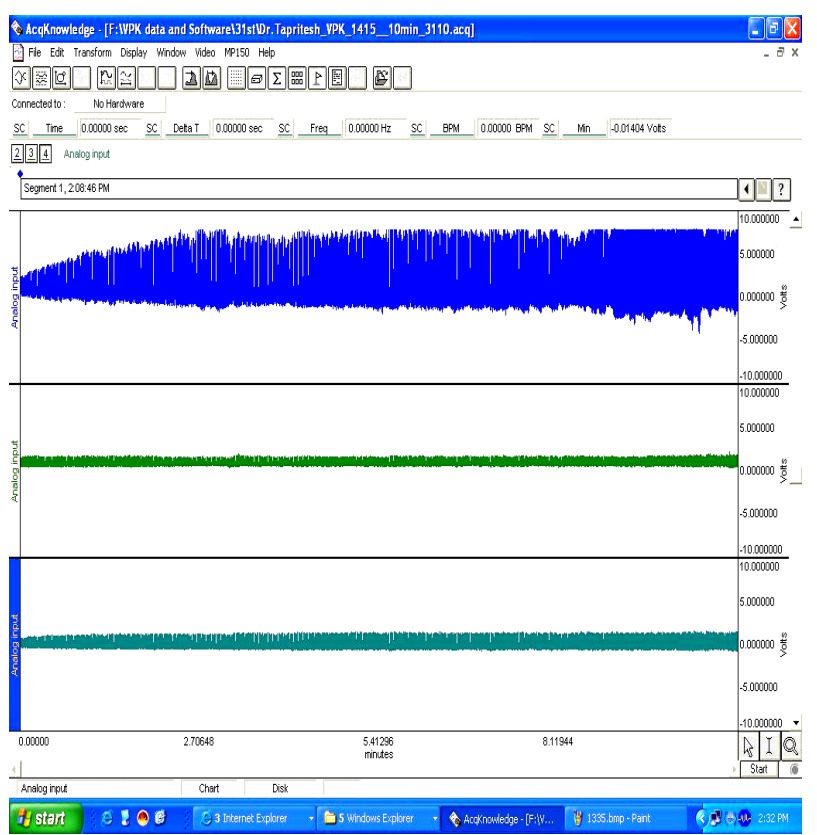

Figure 17. Signals captured at 1355 hrs.

of power in the $1^{\text {st }}$ channel increases as appetite increases from 1315 to 1355 hrs and then falls post lunch at 1755 hrs. Thus, the dynamic properties of the three signals (magnitude of power and frequency information) change before lunch and after lunch. Further, a $30 \mathrm{~min}-$ ute post-lunch signal (1705 hrs) was divided into 6 segments of 5 minutes each and PSD for every segment was calculated. It can be inferred that the magnitude of power in the $2^{\text {nd }}$ channel decreases and that of the $3^{\text {rd }}$ channel increases over time.

A post-lunch 30 minute signal was divided into 15 segments of 2 minutes each and bandpower calculated 


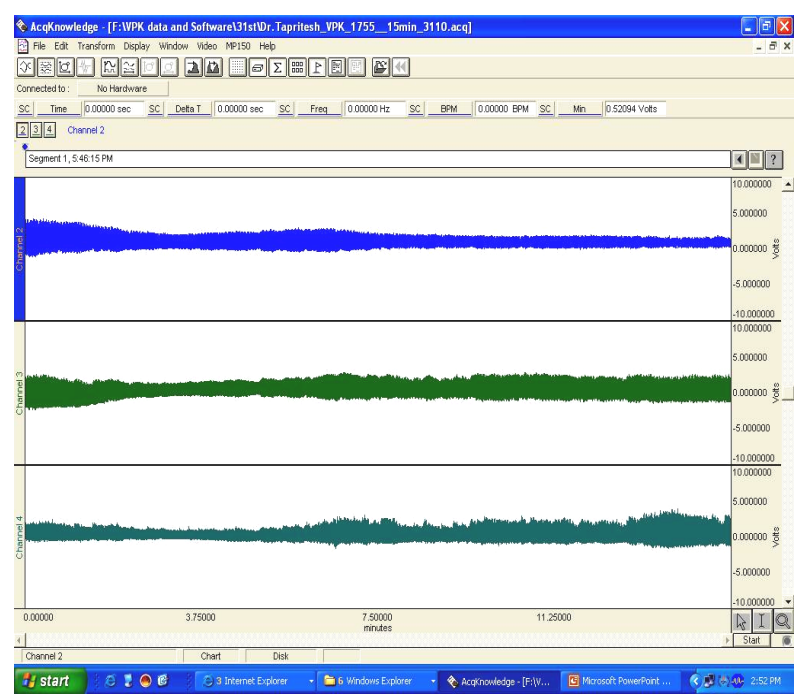

Figure 18. Signals captured at 1705 hrs.

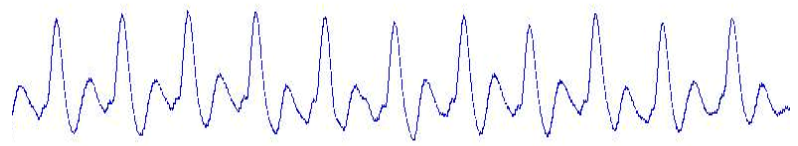

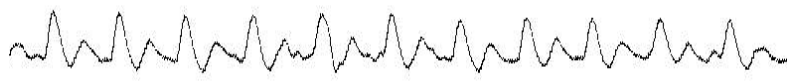

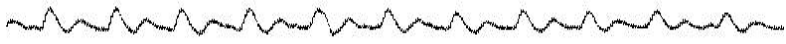

Figure 19. Zoomed view of a portion of signals from Figure 10.

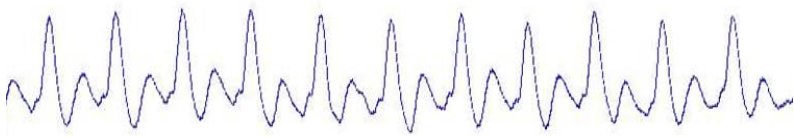

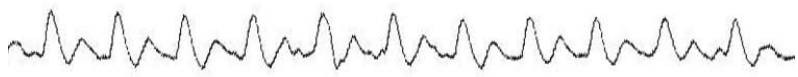

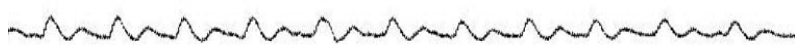

Figure 20. Zoomed view of a portion of signals from Figure 12.

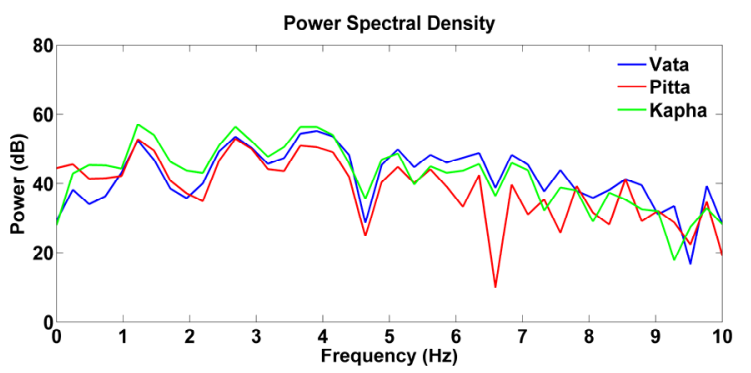

Figure 21. PSD of the signal acquired before lunch at $1315 \mathrm{hrs}$.

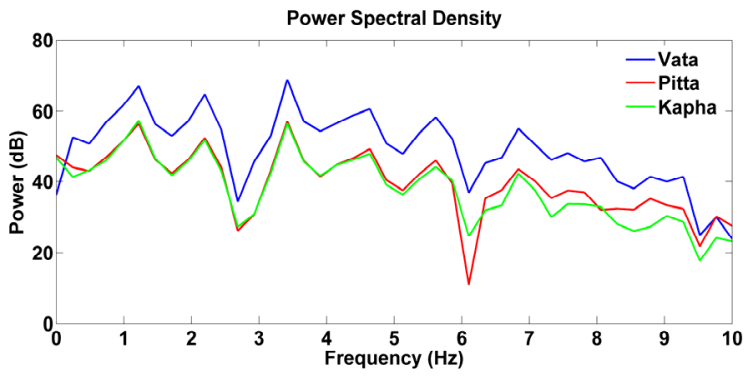

Figure 22. PSD of the signal acquired before lunch at $1355 \mathrm{hrs}$.

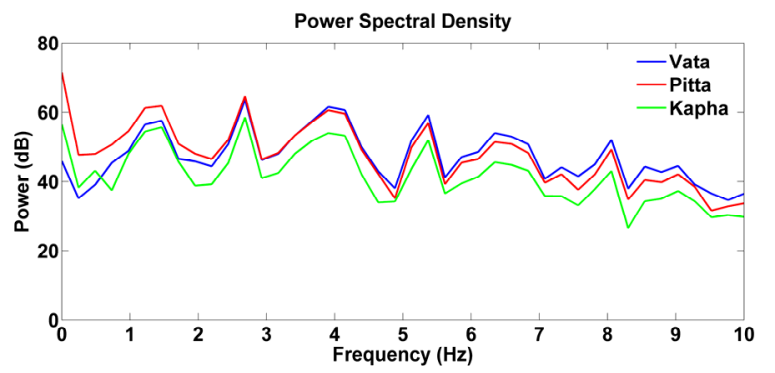

Figure 23. PSD of the signal acquired after lunch at 1705 hrs.

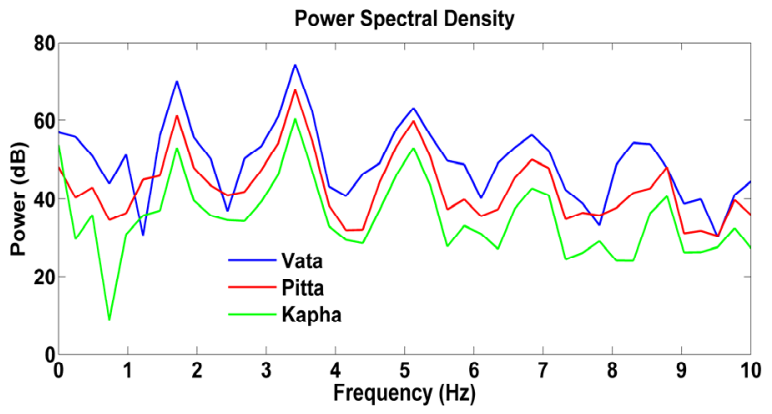

Figure 24. PSD of a post-lunch signal divided into 6 segments.

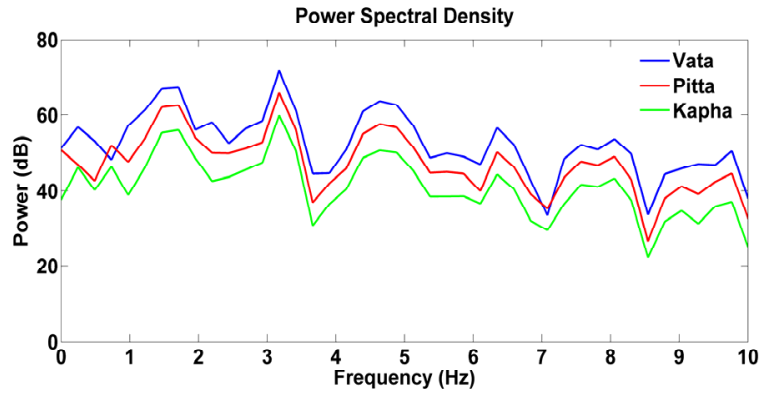

Figure 25. PSD of a post-lunch signal divided into 6 segments. 


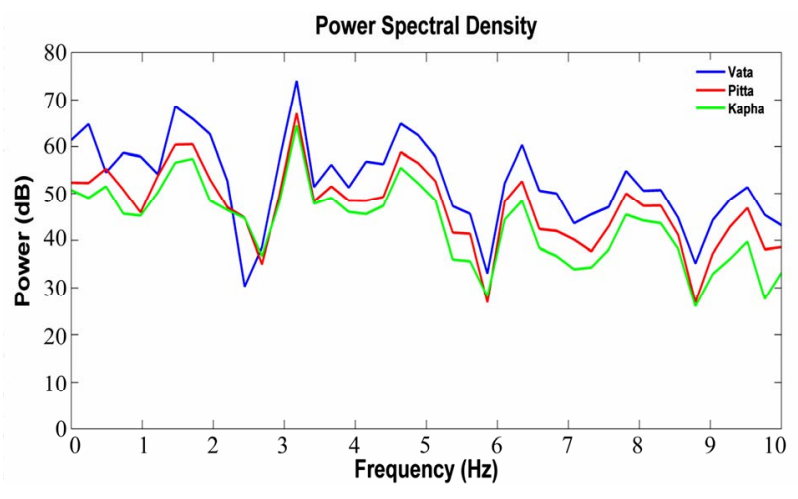

Figure 26. PSD of a post-lunch signal divided into 6 segments.

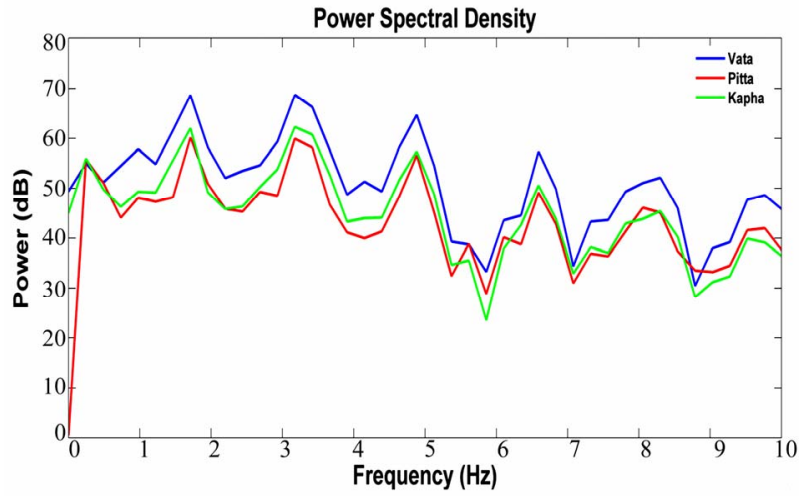

Figure 27. PSD of a post-lunch signal divided into 6 segments.

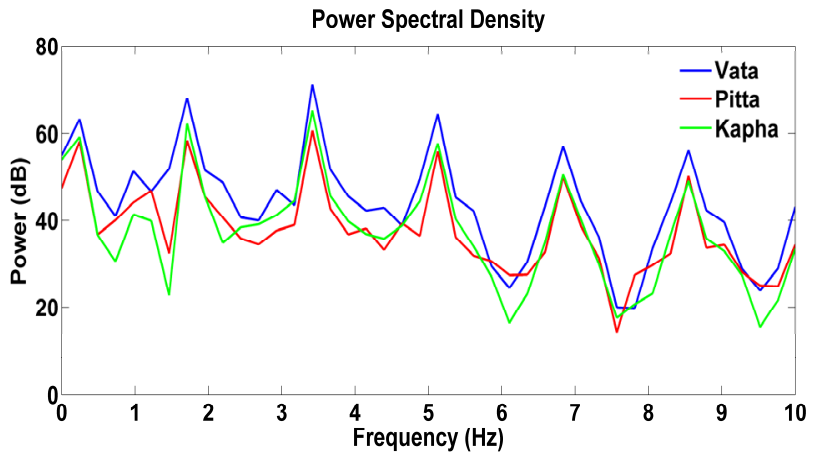

Figure 28. PSD of a post-lunch signal divided into 6 segments.

for each segment. The calculated bandpower is the area under the curve of a PSD in the bandlimits $0.5-10 \mathrm{~Hz}$, appropriate for arterial pulse signals. It can be seen from Figure 30 that bandpower for the $2^{\text {nd }}$ channel falls after lunch whereas bandpower for the $3^{\text {rd }}$ channel increases. Bandpower for the $1^{\text {st }}$ channel remains predominant.

Spectral centroid frequency commonly referred to as median frequency was also calculated. It can be seen from Figure 31 that the centroid frequency of the $2^{\text {nd }}$ channel is higher than the $1^{\text {st }}$ and the $3^{\text {rd }}$ channels.

Thus, it is seen that dynamics of the radial pulse signal change on application of stimulus for example administration of lunch. These dynamics can be monitored using the device developed.

\section{CONCLUSIONS}

There is enough evidence in ancient literature that there is not a single disease in the human body which cannot be diagnosed by examining the pulse. However, ancient medical practitioners had to totally rely upon years of clinical experience in order to come to any conclusive diagnosis. Clinicians today have limited examination of the pulse to its rate, rhythm and volume by virtue of which they hardly come to a concrete diagnosis. If there could be a system by which the radial pulse could be critically examined, it could be one of the most useful tools in the field of non-invasive diagnosis of disease. In this paper, such a system known as Nadi Yantra has been developed. Further, wavelet based techniques were used to decompose the pressure signal from the radial artery. Multi-resolution wavelet analysis was used to detect the percussion peaks and the P-P time series was obtained. A HRV plot depicting function of the ANS was obtained

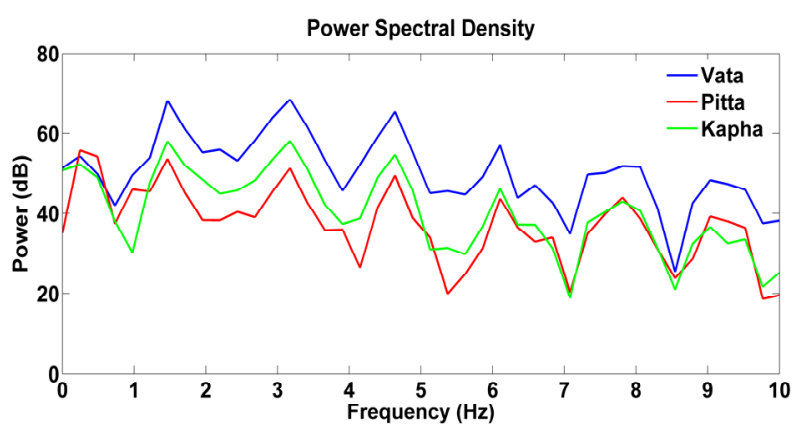

Figure 29. PSD of a post-lunch signal divided into 6 segments.

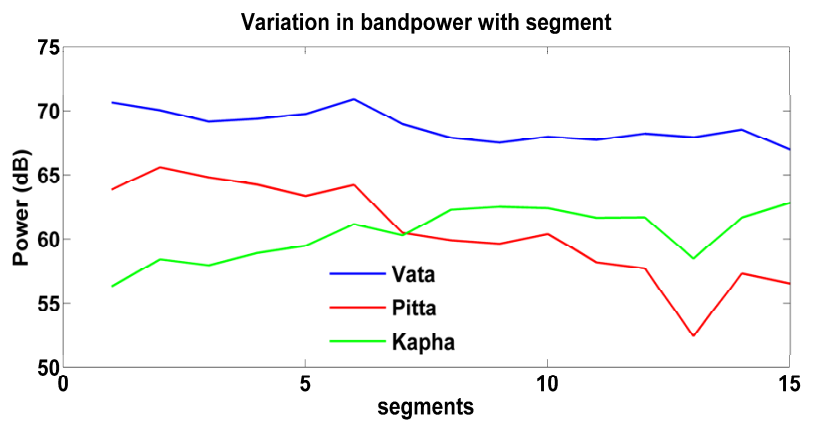

Figure 30. Variation in band power with segment.

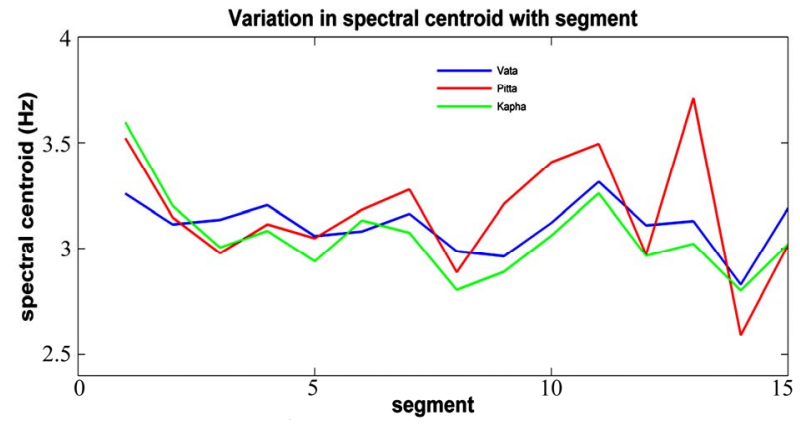

Figure 31. Variation in spectral centroid with segment. 
from the P-P time series. Time domain and Spectral analysis of the P-P series gave significant features. Non-linear Poincare analysis was carried out to obtain a relationship between consecutive P-P intervals. These features hold significance in the study of short term and long term variability of the PP time series. Wavelet based method proved most effective because of its ability to filter out noise and low frequency components and retain relevant detail. The wavelet based detector has advantages of time-scale analysis as well as frequency analysis. This approach to capture the peaks from the radial artery and further, extraction of features from the $\mathrm{P}-\mathrm{P}$ series is a promising tool for studying the diagnostic application of Nadi Yantra in the assessment of the autonomic nervous system. The experiments described in the paper show that Nadi Yantra has the potential to objectively measure and display the physiological changes occurring in the human body.

\section{ACKNOWLEDGEMENTS}

We would like to extend our thanks to Dr. Mitali Mukerji, Dr. Bhavana Prasher, Dr. Shilpi Aggarwal and Dr. Tavpritesh Sethi from the Institute of Genomics and Integrative Biology, New Delhi and Mr. Nandakumar Selvaraj from CBME-IIT Delhi for their support and encouragement. We would also like to thank Prakhar Prakash for his help with wavelet decomposition.

\section{REFERENCES}

[1] Yoon, Y., Lee, M., and Soh, K., (2000) Pulse type classification by varying contact pressure, IEEE Engineering in Medicine and Biology Magazine, 19, 106-110.
[2] Fuzzy Theory in Traditional Chinese Pulse Diagnosis, Proceedings of 1993 International Joint Conference on Neural Networks.

[3] Wang, H. and Cheng, Y., (2005) A quantitative system for pulse diagnosis in traditional chinese medicine, IEEE EMBS, 5676-5679.

[4] Xu, L. S., Wang, K. Q., and Zhang, D., (2002) Modern researches on pulse waveform of TCPD, IEEE.

[5] Joshi, A., Kulkarni, A., Chandran, S., Jayaraman, V. K., and Kulkarni, B. D., Nadi tarangini: A pulse based diagnostic system, Proceedings of the 29th Annual International Conference of the IEEE EMBS.

[6] Grap, A., (1995) An introduction to wavelets, IEEE Comp. Sc. and Engg., 2(2).

[7] Torrence, C. and Compo, G. P., (1998) A practical guide to wavelet analysis, American Meteorological Society.

[8] Holger, G. and Adelmann, (1999) Design of a PC-based system for time-domain and spectral analysis of heart rate variability, Computers and Biomedical Research, 32, 77-92.

[9] Task Force of the European Society of Cardiology and The North American Society of Pacing and Electrophysiology, (1996) Heart rate variability standards of measurement, physiological interpretation and Clinical use, European Heart Journal, 17, 354-381.

[10] Malik, M., (1996) Heart rate variability-Standards of measurement, physiological interpretation and clinical use-Task Force-European Society of Cardiology and The North American Society of Pacing and Electrophysiologu, Eur. Heart J., 17(354), 81.

[11] Brennan, M., Palaniswami, M., and Kamen, P., (2002) Poincare plot interpretation using a physiological model of HRV based on a network of oscillators, Am. J. Physiol. 283: H1873-H1886. 